\title{
A Review of Bluetooth based Scatternet for Mobile Ad hoc Networks
}

\author{
Khizra Asaf*, Muhammad Umer Sarwar ${ }^{\dagger}$, Muhammad Kashif Hanif ${ }^{\ddagger}$, Ramzan Talib ${ }^{\S}$, and Irfan Khan ${ }^{ף}$ \\ Department of Computer Science, \\ Government College University, Faisalabad, Pakistan
}

\begin{abstract}
Bluetooth based networking is an emerging and promising technology that takes small area networking to an enhanced and better level of communication. Bluetooth specification supports piconet formation. However, scatternet formation remains open. The primary challenge faced for scatternet formation is the interconnection of piconets. This paper presents a review of the proposed approaches and the problems confronted for establishing scatternet for ad hoc networks specifically MANET. In this work, a comparison of the Blue layer algorithm with MMPI interface based algorithm on Bluetooth scatternet formation. The enhancement in the developed MMPI framework makes it a good option for scatternet applications.
\end{abstract}

Keywords-Bluetooth; ad hoc network; piconet; scatternet; MANET

\section{INTRODUCTION}

Bluetooth is a basis for transformative wireless ad hoc connectivity. It is among the technologies that can serve as a communication tool even when cellular network is not working or destroyed. Bluetooth permits the devices to make a short range network called piconet. Piconet has the capability to support up to eight bluetooth devices. One device takes the master device role and the other devices become the slave. The network topology resulted by the interconnection of piconets is known as scatternet. Bluetooth standard permits multiple tasks for the same node. It can act both as a master in one piconet and slave in even more than one piconet at the same time.

In last decade, traditional cell phones are upgraded to smart phones at a remarkable rate. This noticeable up-gradation is due to the substantial increase in functionality of the smart phones. Mobile applications are playing a vital role for the betterment of the world in almost every field of life. The applications are designed and developed with the deliberation for the need and demand of time. Today, everyone is familiar with the natural incidents as well as with the planned attacks. Thus, it emerges to focus on more efficient ways for personal safety and assistance. In this regard, communication technologies and mobile applications have played a vital role.

Mobile wireless technology is flagging the way for mobile ad hoc networks and bluetooth is supposed to be a reasonable option. It empowers and supports multi-hop communication. Scatternets are the best way to achieve high data transfer capacity by forming as many piconets as conceivable in a scatternet. Indeed, scatternets are considered as a preferential and recommended topology for establishing a point to the multi point piconet.
Different applications can be hosted and presented for one hop communication using bluetooth technology. However, scatternet formation is a problem that has still not been dealt yet. Bluetooth based scatternet formation faces some technical difficulties such as routing, interlinking piconets, and scheduling. Routing is suggested as one of the procedural issues in scatternet formation. Nodes can connect and disconnect with the network at random times. This feature of node mobility can create problems in network enactment. Moreover, bluetooth specification does not provide any particular protocol for establishing scatternet. The problem of scatternet formation turns to be significantly difficult if the starting nodes have no acquaintance with their ambiances [1]. The process of encountering network is a frequency hopping structure; nodes in immediacy must coordinate among frequency hopping configurations and timing before being available for the communication mode.

Mainly two processes are defined for building a scatternet: 1) piconets should be interlinked to form a network; and 2) the bridge nodes must maintain connectivity between piconets. The remaining paper is organized as: Background information on Bluetooth based mobile ad hoc networks is presented in Section II. Related work is described in Section III. Section IV compares compares Bluelayer algorithm and MMPI interface algorithm. Finally, the paper concludes the outcomes in Section $\mathrm{V}$.

\section{BACKGROUND}

\section{A. Mobile ad-hoc network (MANET)}

Ad-hoc networks have been a fascinating and worth considering research zone for almost two decades. A Mobile Ad-hoc network commonly known as MANET is a developing wireless network. Mobile nodes associate on the ex-temporal basis and are considered as self-forming as well as self-healing network. This feature enables peer-level dynamic infrastructure between mobile nodes. Fig. 1 shows an ad-hoc network formed by the interconnection of piconets.

In ad hoc network, each node uses the same station to execute multi-hop progressing. Each node plays the role of a host and a router to path the packets to and from nodes beyond the communication range [3]. The unusual aspects of MANET empower it to provide major assistance's in an environment of vibrant structure formation.

\section{B. Piconet}

Bluetooth devices connect in an ad hoc manner and form a network called piconet. A piconet may consist of minimum two devices. This number can be extend up to a maximum of eight 


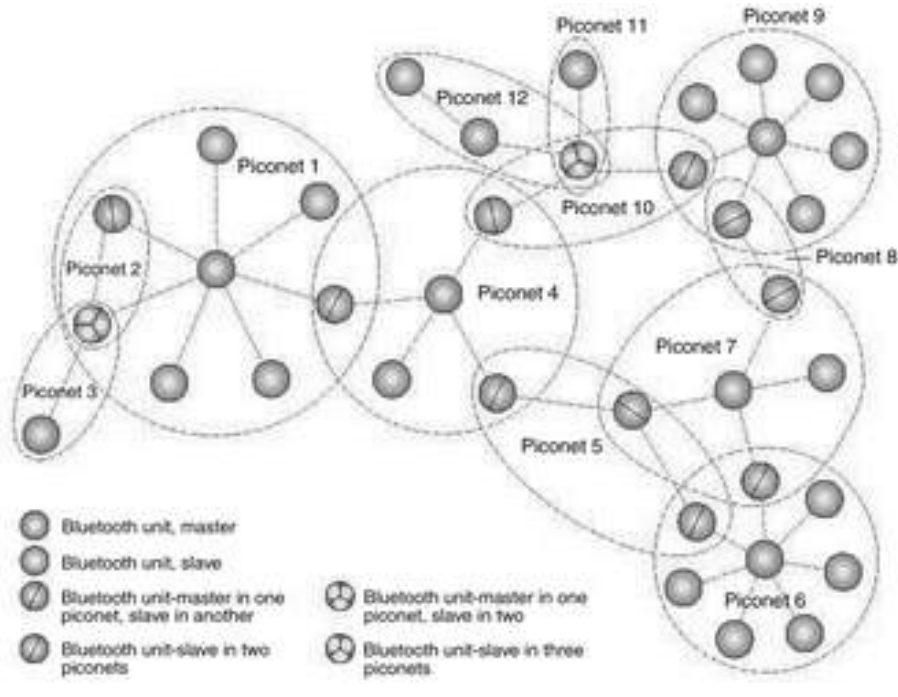

Fig. 1. Mobile ad hoc network [2].

devices. Fig. 2 shows a piconet formed by the combination of a single master and its slaves. In a piconet, one member will act as a master while the other members act as slave in the network. Every piconet has a single master and hops individualistically. The unit establishing piconet is a master and the devices discovered are the slaves. The hopping classification is decided by the master of the particular piconet. In point of fact, the limit of slaves connected to a master is applicable for active devices not for connected devices. Only seven slaves can be active at the same time. The remaining connected devices have to be in a state called parked state. Piconet can be single slave and multi-slave. Single slave piconet has only one slave while multi-slave piconet has many slaves but not more than seven.

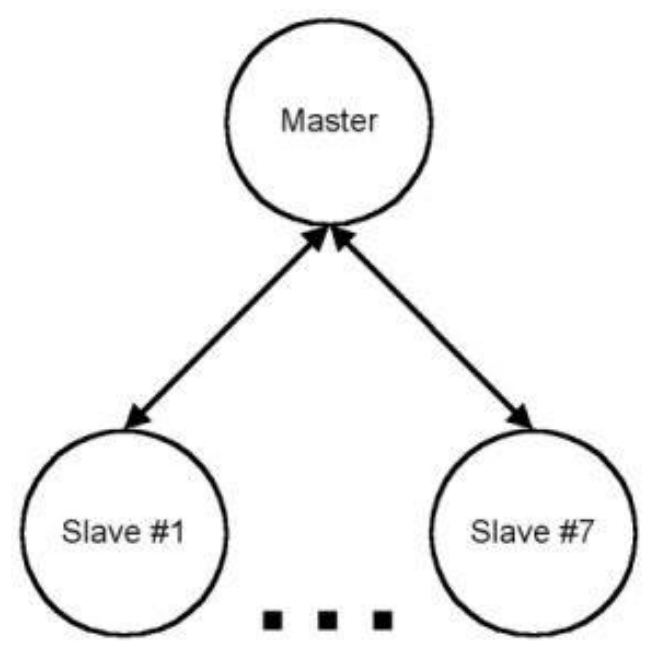

Fig. 2. Piconet with a master and seven slaves [4].

\section{Scatternet}

Scatternet is a network of interconnected piconets. This type of interlinked network can increase networking tractability and expedite new applications. Fig. 3 depicts a scatternet formed by interconnection of the two piconets. A peer to peer connection is created in scatternet. It is formed when a member of one piconet behaves as a slave in another piconet. A single device is permissible to act as a bridge for numerous piconets [5]. The participating member can either be a master or a slave. A device can perform as a slave device in more than one piconet. However, it can act as a master device only in one piconet. The device existing in two piconets works as transmit device. This intermediate device forwards data packets among the piconets using the concept of time division multiplexing (TDM) or time sharing. Point to multipoint competency is required for the device to be a part of scatternet communication. Scatternet permits communication among more than eight devices thus increasing the number of devices in a network. It makes intelligent use of bandwidth.

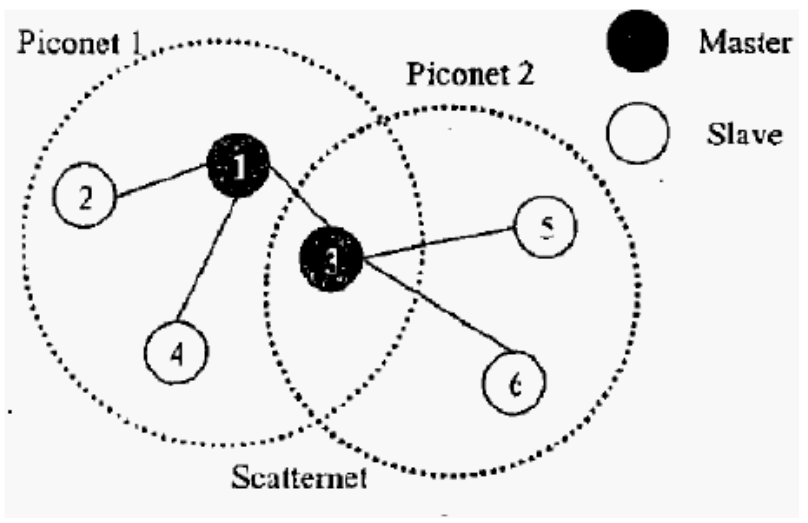

Fig. 3. Scatternet formed by overlapping of piconets [6].

\section{RELATED WORK}

Different researchers have proposed various algorithms for scatternet formation. The way piconets are interlinked and the method for formation of a scatternet significantly affect performance. Numerous topology establishment algorithms are suggested for creating multiple ad hoc networks. Scatternet models have classified as tree-based or mesh topology. Tree structure allows a single route for each node pair whereas mesh topology allows multiple paths between the nodes. The algorithms are classified into two categories [7]. One deals with the restriction of all the nodes in the radio range and other deals with both types of nodes; within and outside the radio range.

\section{A. Tree Topology}

Different approaches exists for tree building algorithms. Tree scatternet formation (TSF), SHAPER, and SFX are mostly used. Multi-hop scatternets form a tree-like structure. The term tree compromises the master-slave linkage. TSF is a simple algorithm for building a tree-shaped structure. The root of the tree becomes the master. In TSF, all the nodes of the network lies within the communication range and can communicate with each other. TSF targets at dynamic situations. The connectivity, communication efficiency and healing power enrich the working of TSF algorithm [8].

Considering the SHAPER algorithm for scatternet formation is a more genuine and convincing approach. It is a 
distributed self-healing algorithm which offers QoS support. SHAPER is the first flexible algorithm among scatternet formation algorithms. It can adapt and adjust the topology in a multi-hop state according to the mobility and disasters of nodes [9]. SHAPER works for the nodes even when they are not in the bluetooth radio range. This algorithm provides the network with self-healing capability in case of any trouble.

SFX was built using SHAPER algorithm by extending the realistic conditions to a coherent and corroborated explanation. SFX was proposed with the aim to develop and uphold a bluetooth node tree with an appropriate organization of piconets. The merging technique in SFX is exceedingly asymmetrical and is a significant peculiarity to SHAPER algorithm [10]. SFX builds a parallel structure and permits any number of trees to merge with the same tree at the same time. The principal idea of SHAPER was implemented for SFX. However, some of its features were ignored to avoid the unnecessary complexity. In SHAPER, four distinct merge processes were involved. However, SFX consists of only a single procedure to be handled making it much easier to implement.

\section{B. Mesh topology}

Mesh topology is based on tree shaped structure. The tree shaped structure is generated initially which is converted to mesh shaped topology. The mesh topology was proposed to decrease the route lengths of a standard tree to a sufficient extent [7]. The backward connection mechanism is used in this topology to create multiple paths among the nodes of the network. Master/Slave Web (MSW) and Slave/Slave Web (SSW) coordinate to generate a mesh scatternet.

MTSF is a mesh topology scatternet formation algorithm presented for the establishment of a scatternet. MTSF was capable of managing addition and deletion of nodes in a network [11]. It removes the restriction of bluetooth radio range. Thus, building a larger scatternet. MTSF has three phases. In the initial phase, nodes get to know their neighbors. This period is called the discovery phase and is common in almost all the algorithms. Clusters of piconets are formed in second phase. The piconets are interlinked in the third stage.

BlueMesh is a protocol for scatternet establishment. It forms a mesh of devices having diverse paths between nodes. BlueMesh does not restrict the bluetooth devices to be available in the broadcast range for scatternet formation. It is found to be operational and quick regarding scatternet generation. Furthermore, it provides the benefit of comparing the shortest route among multiple paths between any two member nodes. BlueMesh consists of two phases. The first phase is device discovery phase and the second phase is responsible for forming piconets and interconnecting the piconets to form a scatternet [12].

\section{COMPARISON}

This section presents the comparison of BlueLayer and Mobile Message Passing Interface algorithms.

\section{A. BlueLayer}

BlueLayer diminishes complications of topology establishment outlays. This algorithm was designed in a way to take benefits of tree shaped and mesh shaped topologies [13]. In BlueLayer, a web-shaped topology is formed. It can identify new roots during the phase of scatternet formation. Every new source creates and possesses a confined web shaped sub-net by coordinating with other roots. Three pre configured network factors are assumed to generate separate web shaped sub-nets.

(a)Scatternet Formation Procedure;

Initialization:the first root sets $\mathrm{k} 1=\mathrm{k}^{*} \mathrm{v}$,connects up-to 7

slaves and generates new masters;

if a downstream master is connected, then

$k_{1}=k_{1}-1$, each new master propagates $\left(k_{1}, k, v\right)$

in its downstream direction ;

if $k_{1}=0$, the $k_{1}^{\text {th }}$ master becomes a new root then

$v=v+1$ and $k_{1}=k * v$, the new root

propagates $\left(k_{1}, k, v\right)$ in its downstream

direction and executes a return connection

procedure for the first root;

if a leaf node is connected then

the leaf node starts the return connection procedure for its upstream roots to build their own sub-nets;

else

each node continuously propagates

$\left(k_{1}, k, v\right)$ in its downstream direction end

end

end

end

(b)Return Connection Procedure;

Initialization:The leaf master node propagates an initial return variable $r=1$ in its upstream direction;

if the upstream master is reached then

$r=r+1$ until the immediate upstream root is reached;

if $r>k_{1} / 2$ at its upstream root node then

the root retains its role as a root and gather nodes information of its downstream master until all its downstream masters notifications as well as the algorithm stops;

else

the root switches its role to a master and deliver its affiliated information to its upstream root

end

end

end

Algorithm 1: The Pseudo code of Bluelayer scatternet formation

Scatternet shape significantly influences routing protocol strategy. Therefore, the tier-ring addressing scheme has been presented to attain self-routing protocol. This protocol comprises of two phases; address query phase and a forwarder decision phase. First phase trace and pinpoint a destination and the second phase decide the ideal master as a forwarder.

\section{B. Mobile Message Passing Interface}

Mobile Message Passing Interface (MMPI) is a library presented for implementing the functionality of MPI on bluetooth empowered mobile devices. It offers point to point and global communication. The library was designed mainly for 


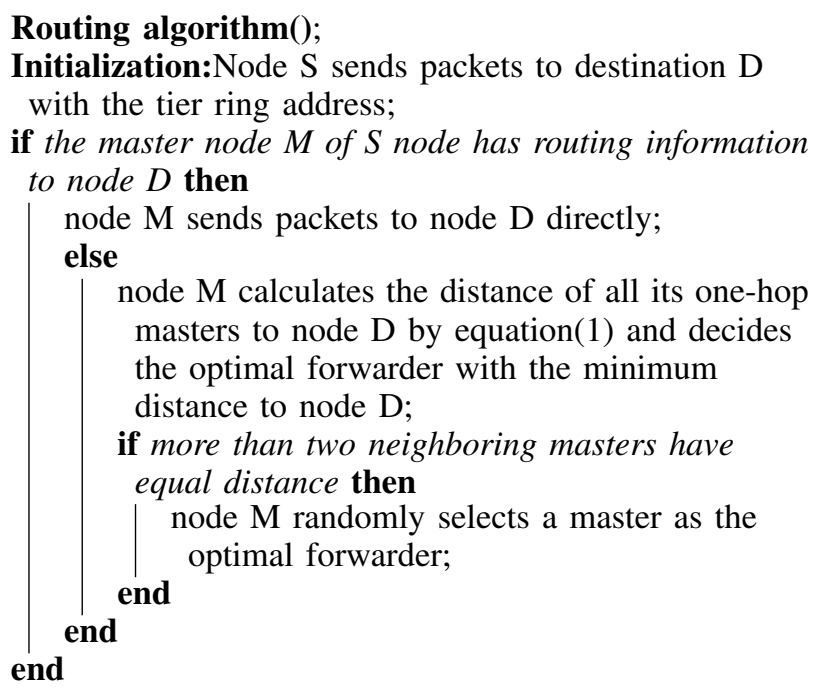

Algorithm 2: The Pseudo code for Forwarder decision

piconets. A solution was presented that provided the same functionality as offered by MMPI library and was implemented over a Scatternet [14]. For scatternet formation, a significant advancement of the MMPI library is required to organize the network setup as well as to permit intercommunication of the devices. The enhancement made in the library encompasses some distinctive modules while holding all of its unique functionality. MMPI node and CommsCenter are among the significant parts of the enhanced MMPI library. The CommsCenter class is considered as the heart of the library as it takes raw information and interprets it into MPPI messages. The MMPI node level delivers the interface between the MMPI class and the CommsCenter. This class is also responsible for device discovery process at the initial level. With this, the interface to the library is the MMPI class. Data is provided through a defined order that is started at the CommsCenter and lasting up to MMPI class.

Data: list of all MMPI capable devices

Initialize list of devices to send to bridge node;

foreach deviceinthelist do

create master connection;

add bridge node to routing table;

else if add node to routing table then

create slave connection;

add slave node to routing table;

send slave message;

else if after bridge node then

add device to list of devices to send to

bridge node;

add node to routing table;

$$
\text { end }
$$

end

end

add number of devices to bridge message;

add list of devices to bridge message;

Algorithm 3: Establishing initial connections at the root node

Scatternet formation is initiated at the root node. First, an inquiry is performed for the devices offering MMPI provision.
Then, the root node selects the number of piconets vital to support the scanned MMPI nodes. A bridge node is selected among the network and a list of the addresses of the nodes are sent to bridge node by the root node. The bridge node then selects the master of the second piconet and send it the list of the other devices. The master of the second piconet then establish connections with each device of the list to form a scatternet. Message routing system broadcasts a validation message. A message routing table is used to send a message through the network. Every node has a table of all other nodes and the nodes are routed using the provided index.

Data: list of devices sent by root node

Initialize list of devices to send to additional master;;

foreach deviceinthelist do

if second master then

create master connection ; add second master to routing table;

end

else

| add node to routing table; end

end

add number of devices to Master message;

add list of devices to the Master message;

send Master message;

Algorithm 4: Establishing connections at the bridge node

The idea of the scatternet is an integral as well as a required part of Bluetooth aspect. It is suggested as the best approach to get high data broadcast capability. Scatternet is indeed a preferred way for point to multipoint communication and fulfills the requirement of MANET in an accepted way. Keeping in view the components required for scatternet formation, Bluelayer algorithm is a good approach as it exhibits good network scalability and routing competence for scatternet. On the other side, MMPI library is capable of supporting ad hoc networks of more than eight devices using the scatternet framework.

\section{Conclusion}

Bluetooth specification facilitates piconet formation. Conversely, it does not provide any answer for scatternet development. Blue layer proposes an approach to alleviate the communication as well as computation disbursements for forming a scatternet. It capably creates various sizes of topology formations thus achieving scalable network along with good routing adeptness. On the other side, enhancement in the MMPI library makes it adept and proficient for parallel computing on ad hoc networks. The improvement in the developed framework alleviates the limitation of node thus making it available for scatternet applications.

\section{REFERENCES}

[1] T. Salonidis, P. Bhagwat, L. Tassiulas, and R. LaMaire, "Distributed topology construction of bluetooth wireless personal area networks," IEEE Journal on Selected Areas in Communications, vol. 23, no. 3, pp. 633-643, 2005.

[2] "wirelessadhocnetwork," [Online; accessed May 27, 2017]. [Online]. Available: http://www.technologymedia.co.th 
[3] A. K. Sanghi, D. Singh, and H. Rathore, "A study of wireless environment by means of multiple wireless nodes," International Journal of Computer Applications on Wireless Communication and Mobile Networks, no. 7, pp. 31-35, 2012.

[4] "Piconet," [Online; accessed May 27, 2017]. [Online]. Available: http://www.edgefxkits.com

[5] M. Medidi and A. Daptardar, "A distributed algorithm for mesh scatternet formation in bluetooth networks." in International Conference on Wireless Networks. Citeseer, 2004, pp. 295-301.

[6] "Bluetooth devices forming a scatternet out of two piconets." [Online; accessed May 27, 2017]. [Online]. Available: www.researchgate.net

[7] C.-M. Yu, S.-K. Hung, and Y.-C. Chen, "Forming mesh topology for bluetooth ad hoc networks," in Consumer Electronics (ISCE), 2013 IEEE 17th International Symposium on. IEEE, 2013, pp. 123-124.

[8] A. OLUWARANTI, "Evaluation of tree scatternet formation algorithm for enhanced bluetooth scatternet," Journal of Sustainable Technology, vol. 2, no. 2, pp. 28-36, 2015.

[9] F. Cuomo, T. Melodia, and I. F. Akyildiz, "Distributed self-healing and variable topology optimization algorithms for qos provisioning in scatternets," IEEE Journal on Selected Areas in Communications, vol. 22, no. 7, pp. 1220-1236, 2004.
[10] M. Methfessel, S. Peter, and S. Lange, "Bluetooth scatternet tree formation for wireless sensor networks," in Mobile adhoc and sensor systems (MASS), 2011 IEEE 8th international conference on. IEEE, 2011, pp. 789-794.

[11] S. Sunkavai and B. Rarnalmurthy, "Mtsf: a fast mesh scatternet formation algorithm for bluetooth networks," in Global Telecommunications Conference, 2004. GLOBECOM'04. IEEE, vol. 6. IEEE, 2004, pp. 3594-3598.

[12] C. Petrioli, S. Basagni, and I. Chlamtac, "Bluemesh: degree-constrained multi-hop scatternet formation for bluetooth networks," mobile Networks and Applications, vol. 9, no. 1, pp. 33-47, 2004.

[13] C.-M. Yu and Y.-B. Yu, "Joint layer-based formation and self-routing algorithm for bluetooth multihop networks," IEEE Systems Journal, 2016.

[14] B. J. Donegan, D. C. Doolan, and S. Tabirca, "Mobile message passing using a scatternet framework," International Journal of Computers Communications Control, vol. 3, no. 1, pp. 51-59, 2008. 